\title{
Automatic Multi-Operation Machine Using Low Cost Color Sensor and Plc
}

\author{
D. Ajay abilash ${ }^{1}$, P. Kayalvizhi ${ }^{2}$ and C. Ramachandra Durai ${ }^{3}$ \\ Department of mechanical engineering, \\ Periyar Maniammai University, \\ Thanjavur-613403 Tamilnadu, India \\ mechajay.k@gmail.com ${ }^{1}$, kayal.electrocom@gmail.com², \\ ramachandradurai11@gmail.com ${ }^{3}$
}

\begin{abstract}
Sensors play a major role in an automated manufacturing system. They are used for getting information from manufacturing operations and processes that are being performed. They are also used for making decisions about the operations being conducted. The purpose of sensors is to inspect and monitor the work-in-progress interface with the manufacturing equipment. In manufacturing industries the multioperation machine like VMC (Vertical machining center) and HMC (Horizontal machining center) are the major part. It is used to improve the high productivity, high quality and high accuracy of the product. But the CNC machine has some drawbacks like high initial investment, high maintenance and skilled labor wants to operate the machine. To overcome these problems, this paper proposes a low cost automatic multi-operation machine. It performs basic machining operations like drilling, tapping and boring using low cost color sensors (LDR and LEDs). In this system color sensors are the main part to determine the machining operations based on the usage of color LEDs. If LDR detects the color LED the machining operation will take places, the machining operation and job clamping operation are achieved by pneumatic system. All these operations are carried out in a sequence by pre-programming the Programmable Logic Controller.
\end{abstract}

Keywords: Sensors, CNC, Programmable Logic Controller

\section{Introduction}

Industries are basically produce useful goods and services at low cost like low production cost, low machinery cost and low inventory cost [1]. Today's world wants every task to carry out quicker and fast due to technology advancement these advancements also demand huge investments and expenditure. Every industry desires to make high quality of the product at low production cost and also give high productivity at short time period. In an industry a considerable portion of the investment is made for machinery installation [2]. So most of the small scale industries are wants to low cost multi tasking machine in the single system. For the above reason the paper proposes a machine which can perform operations like drilling, tapping, boring using color sensors and also controlled by programmable logic controller.

\subsection{Color Sensors}

Industries today are moving towards the use of color sensors to fulfill their needs for higher production and precise quality. The color sensors components are very expensive and also design is very complicated, so limited application we can use the color sensor. However, new technologies of color sensors with low cost and higher levels of integration are available, for more cost-effective solutions. As the cost of color sensing has come 
down, the number of applications using color sensors is increasing. Color sensors play a major role in much equipment such as color printers and plotters, paints, textiles, cosmetics manufacture and manufacturing application [3]. In this paper, low cost color sensors are used to develop the system. It has three color RGB models which define all primary colors Red, Green and Blue. The sensor consists of a LDR (Light Dependent Resistor) and three color LEDs like red, green, and blue as light source [4]. The LDR and the LEDs are mounted with opposite each other in such a way the radiation from LEDs get reflected to LDR, the resistivity of LDR is inversely proportional to the intensity of color LEDs, the each color LED has different intensity [5]. Therefore, the voltage varies corresponding to different colors. Using this concept, the color LEDs are placed in the system at the turret and work table. The input voltages of two sensors are compared. If the two voltages are equal then the operation takes place in the system.

\subsection{Multi Operation Machine}

The multi operation machine is also called multi tasking machine. It will be able to perform the many no. of operations in one machine like drilling, tapping, boring [6]. It has two rotary motions one is turret (tool holder) and another one is rotary work table. In these two motions are achieved by using drivers like servo motors and it has two axial motion to clamp the object and achieve the machining operation by using pneumatic components like pneumatic cylinder, direction control valve, flow control valve, pressure control valve, mechanical limit switches and compressed air. These components are controlled by programmable logic controller [7].

\section{Problem Description}

All production based industries wants low production cost, high quality in product, high reliability, high accuracy and high work rate which is possible through the utilization of multi-operation machine. But the multi-operation machine (CNC) has a very high initial cost and skilled labor to operate the machine. The fixture design is very complicated in the multi-operation machine (CNC). So this paper proposes the new multi operation machine.

\section{Proposed Methodology}

This system mainly uses the LDR based color detecting sensor to identify the tool for machining the work piece. The system has two rotary parts one is turret (tool holder) and another one is a rotary work table. The turret has three machining tools which are drilling tool, tapping tool and boring tool. The work table has three work piece holders. In the turret three LEDs are placed to emit the light and corresponding one LDR is placed to detect the lights. In the work table the three LEDs are placed to emit the light and one LDR is placed to detect the lights. In the three LEDs are emits the lights in colors of red, green and blue. The red LED is placed near the drilling tool in the turret, the green LED placed near the tapping tool in the turret and the blue LED placed near the boring tool in the turret. Also red, green and blue LEDs are placed near the work piece holder. First, rotary work table starts to rotate using servo motor. If proximity sensor detects the work piece, the rotary table motor gets off and the pneumatic cylinder will extend to clamp the work piece and the turret will start rotating [8]. Then both LDRs give the analog input voltage to Programmable Logic Controller. It will compare the two voltages and give output to the motor which gets switch off and the machining operation will takes place. If both LDR detects red color LED then, the drilling operation will takes place. If both LDR detects green color LED then, the tapping operation will takes place. If both LDR detects the same blue color LED then, the boring operation will takes places. These three 
operations are achieved by compare function using ladder logic language in Programmable Logic Controller [9].

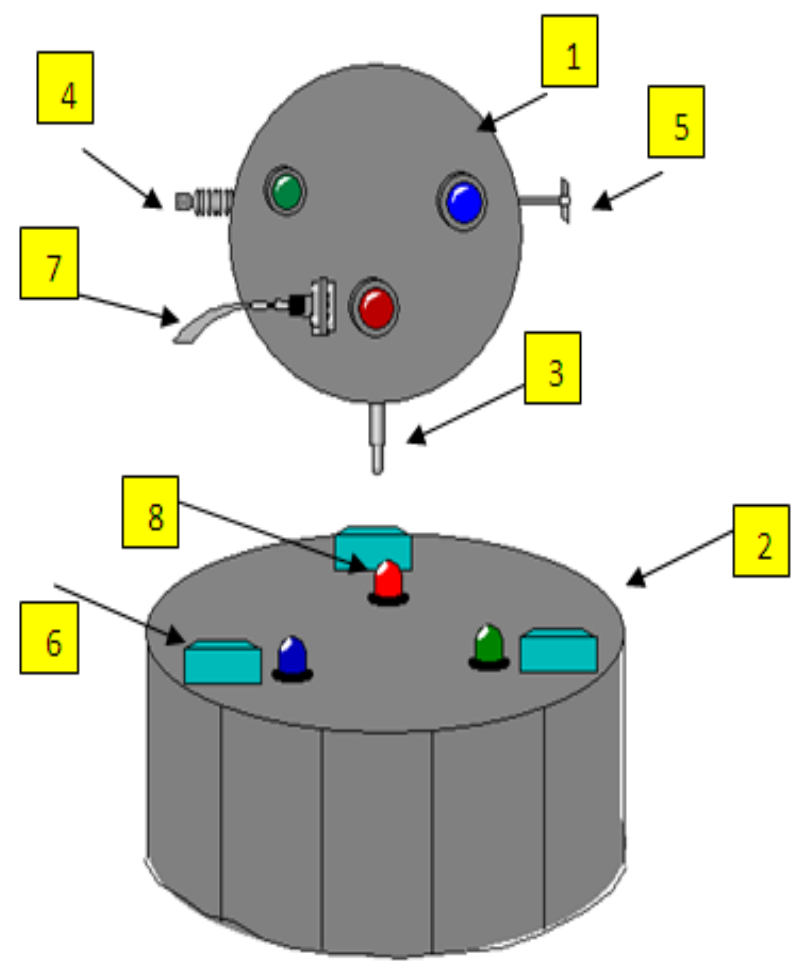

Figure 1. Model of Proposed System

1. Turret assembly

2. Rotary work table assembly

3. Drilling tool

4. Tapping tool
5. Boring tool

6. Work piece

7. LDR

8. LED

\subsection{System Hardware \& Software Description}

The system mainly consists of color sensor, pneumatic system such as cylinders, directional control valve and flow control valve, reed switches, PLC, proximity sensor and motors. In this proposed system pneumatic system are used for clamping and machining the work piece, proximity sensor is used to detect the work piece and it gives digital input signal to PLC, reed switches are used to detect the position of the pneumatic cylinder, Rotary work Table assembly is used to hold the job in the table. The rotary table is coupled with servo motor. The motor is controlled by PLC. The rotary work table has three working chamber to hold the work piece on the table. The three LEDs are placed on the table to control the machining operation, Turret assembly- The turret is a rotary tool holding device. It has three tool holding chamber. The turret is coupled with servo motor. The drilling, tapping and boring tools are placed on the tool holding chamber. And the three red, green and blue LEDs are placed on the turret to identify the tool for correct operation. The red LED is placed near the drilling tool, the green LED is placed near the tapping tool and blue LED is placed near the boring tool. It is used to identify the correct tool for correct work piece, Finally PLC- it is the main element in this system which is used to control the all outputs of the system. It accepts all inputs signal from proximity sensor, reed switches, and color sensors and it control the motors and pneumatic system 
[10]. Siemens s7-200 PLC hardware and step7 micro win software are used in this system.

\section{System Working}

The following steps are system working and sequence of operation for multi-operation machine:

1. Place the work piece on the rotary work table.

2. Press the start push button to start the process.

3. Rotary work table motor will get on, and the work piece moves on the rotary work table with rotational motion.

4. If the inductive proximity sensor detects the work piece, the rotary work table motor will cut off.

5. The cylinder1 will extend to clamp the work piece.

6. The turret motor will start rotating.

7. If both LDRs detect the same color, the turret motor will cut off.

8. If both LDRs detect the red color, from the drilling tool is positioned in the turret to make a drilling operation.

9. If both LDRs detect the green color, from the tapping tool is positioned in the turret to make a tapping operation.

10. If both LDRs detect the blue color, from the boring tool is positioned in the turret to make a boring operation.

11. After a time delay the cylinder 2 extends to achieve the machining operation.

12. If the machining operation is carried out the cylinder 2 retracts to home position.

13. The cylinder 1 retracts to home position.

14. After these steps the process will repeat when the stop push button is pressed. 


\section{Flow Chart}

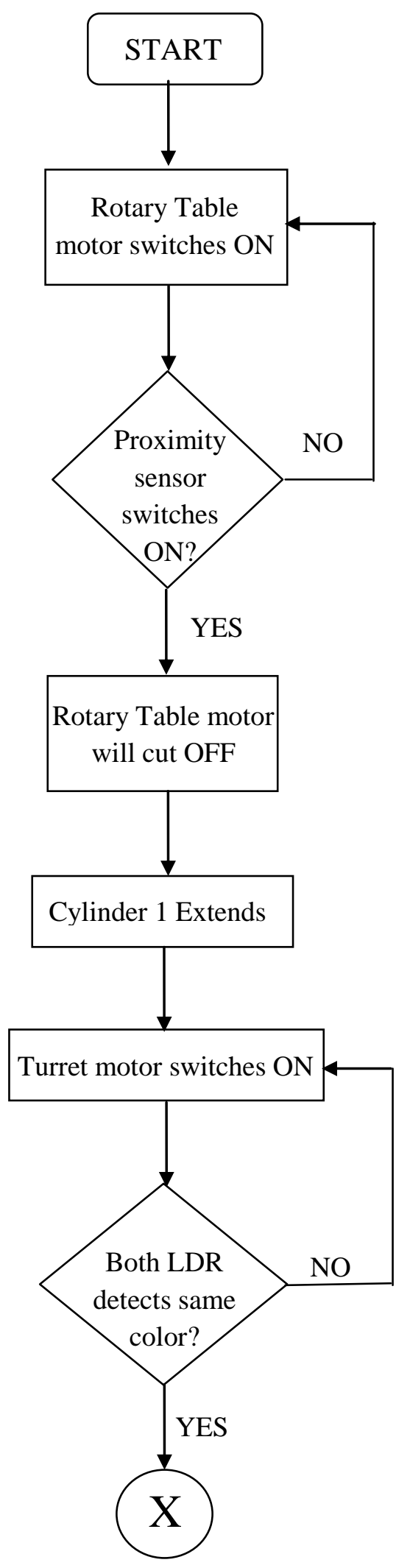




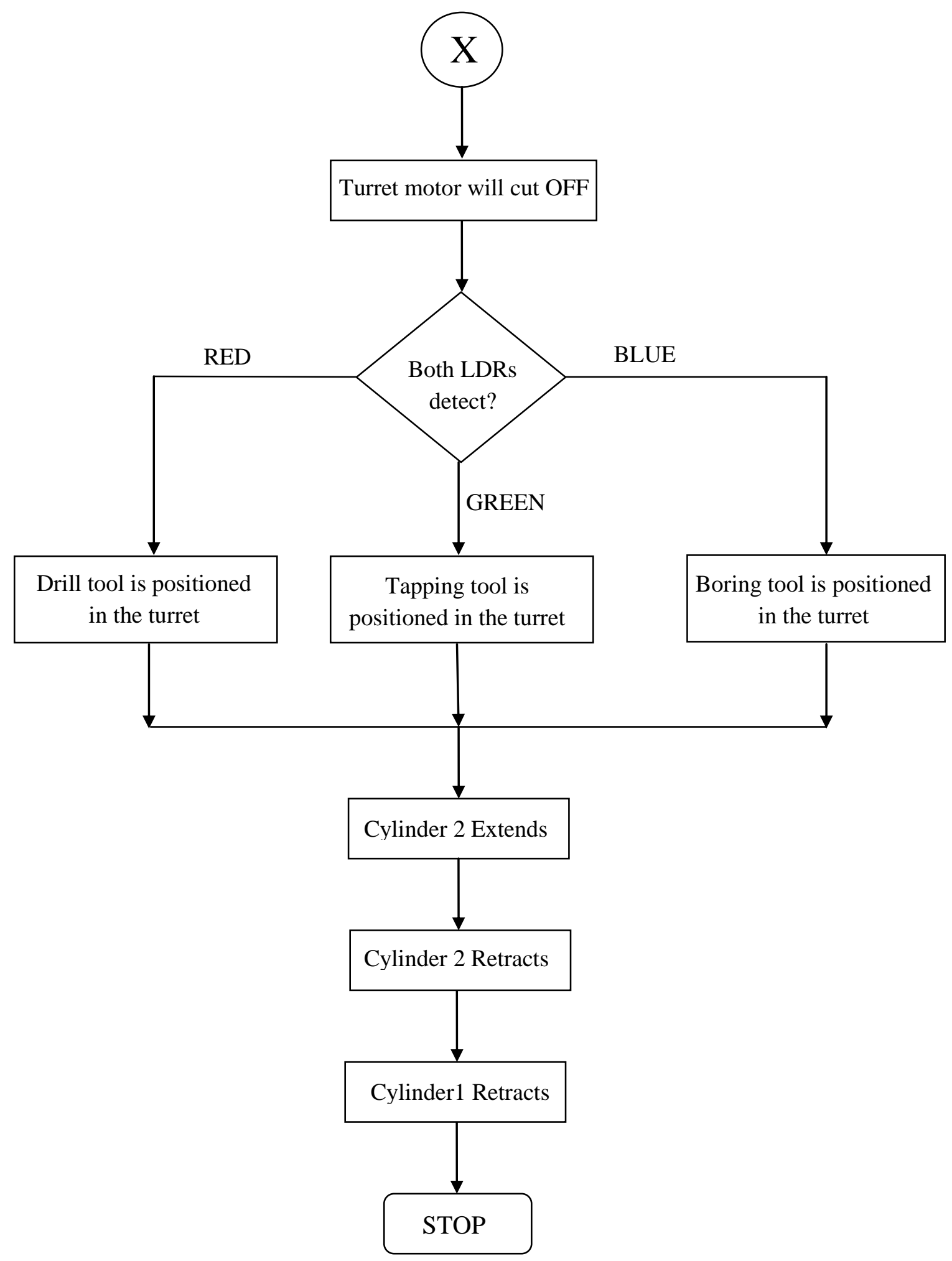

Figure 2. Flow Chart for Proposed System 


\section{PLC Program for Proposed System:}

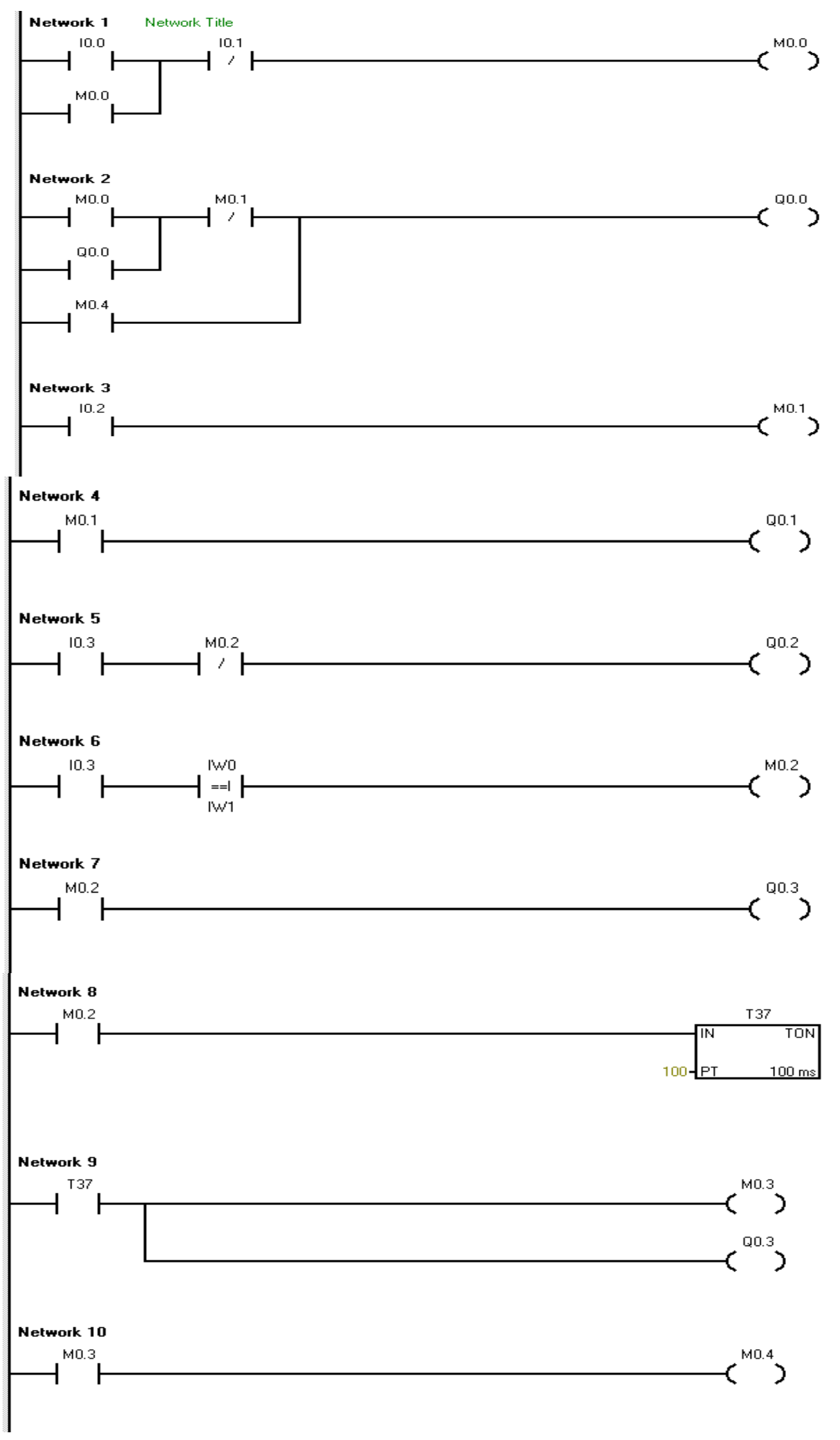




\subsection{Address Assignment in PLC Program}

Table 1. Address Assignment

\begin{tabular}{|c|c|c|}
\hline ADDRESS TYPE & ADDRESS & DESCRIPTION \\
\hline \multirow{4}{*}{ INPUTS } & I0.0 & STOP \\
\cline { 2 - 3 } & I0.1 & START \\
\cline { 2 - 3 } & I0.2 & Proximity sensor \\
\cline { 2 - 3 } & I0.3 & Reed switch \\
\hline \multirow{4}{*}{ OUTPUTS } & Q0.0 & Work table motor \\
\cline { 2 - 3 } & Q0.1 & Cylinder1 \\
\cline { 2 - 3 } & Q0.2 & Turret motor \\
\hline \multirow{4}{*}{ INTEGER } & Q0.3 & Cylinder2 \\
\cline { 2 - 3 } & N7:0 & LDR1 \\
\hline \multirow{5}{*}{ BINARY } & M0.0 & LDR2 \\
\cline { 2 - 3 } & M0.1 & Internal memory 1 \\
\cline { 2 - 3 } & M0.2 & Internal memory 2 \\
\cline { 2 - 3 } & M0.3 & Internal memory 3 \\
\cline { 2 - 3 } & M0.4 & Intemal memory 4 \\
\hline TIMER & T37 & Internal memory 5 \\
\hline
\end{tabular}

\section{Conclusion}

The proposed system is an automatic multi-operation machine which can perform different machining operation like drilling, tapping and boring at the same system using turret and rotary work table. And the all control of the system is achieved by using low cost color sensor (LDR and LEDs), pneumatic components like direction control valve, flow control valve, pressure control valve, pneumatic cylinders, motors and programmable logic controller. Thus in this paper conclude that the multi-operation machine increase the production rate, reduce the machine initial cost, reduce the labor cost because it was a fully automated system and it gives high quality, high accuracy, high reliability.

\section{References}

[1] Muthukkaruppan 1 and Manoj 2, "Low cost automation using electro pneumatic system an online case study in multistation part transfer, drilling and tapping machine", ISARC, (2007).

[2] S. R. Kewate 1, S. V. Karmare 2, N. Sayankar 3 and S. Gavhale 4, "Automatic Speed Control System by the Color Sensor for Automobiles -An Innovative Model Based Approach", vol. 4, no. 2, (2014).

[3] N. B. Daud, "Application of colour sensor in an automated system”, (2007) May.

[4] L. A. Bryan, "Programmable Logic Controllers Theory and implementation", second edition, Industrial Text Company, USA, (1997), pp. 4-104.

[5] I. Mazumdar 1, R. Mondal 2 and S. Pal 3, "Design and Development of a Low Cost Colour Sensor and its application in Angular Position Control System of a Stepper Motor", ISSN 2277-1956/V2N4-12411251, (2010).

[6] S. Srivastava1, S. Srivastava 2 and C. B. Khatri 3, "Multi-Function Operating Machine: A Conceptual Model", vol. 11, Issue 3, Ver. III, (2014) May-June.

[7] Programmable Logic Controller by J. W. Webb and R. A. Reis, (2008). 
[8] D. C. Karnopp, D. L. Margolis and R. C. Rosenberg, "System Dynamics: Modeling and Simulation of Mechatronic Systems", 4th Edition, Wiley, (2006).

[9] L.-L. Wang and H.-Y. Wei, "Development of a distributed control system for PLC-based applications", International Conference on Machine, vol. 2, (2010), pp. 906-909.

[10] Wikipedia, "Light emitting diode", en.wikipedia.org/wiki/Light-emitting_diode.

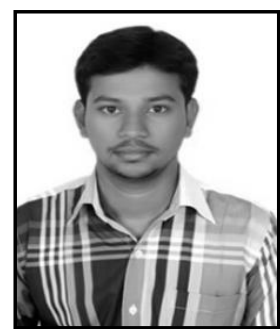

D. Ajay Abilash, He was born and brought up in Thanjavur. I finished my schooling in Kalyana sundaram higher secondary school in thanjavur and I done my UG degree B.E(Mechanical Engineering) in periyar maniammai university situated at thanjavur. Then due to my curiosity in the field of automation now I doing M.tech(Automation \& Control systems) in periyar maniammai university.

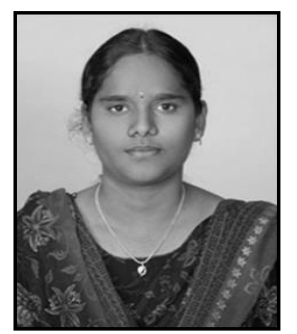

P. Kayalvizhi, She is a native in Thanjavur. I had done my schooling in Sacred Heart Girl's higher secondary school in thanjavur. I got my Under Graduate degree (Electronics and communication Engineering) from Anna University of technology, Tiruchirapalli. Now am doing Post Graduate degree (M. Tech) in Periyar Maniammai University, Thanjavur in the field of automation technology.

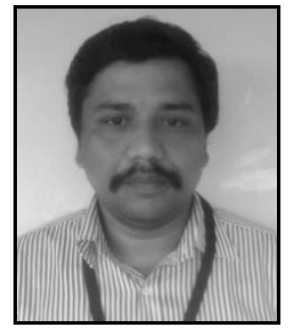

C. Ramachandra Durai, He received my Bachelor's Degree in Mechanical Engineering in 2005 from EGS Engineering College, Anna University, Nagapattinam and Master's degree in Advanced manufacturing system in 2008 from Sastra University, Thanjavur. I am currently working as an Assistant Professor in the Department of Mechanical Engineering in Periyar Maniammai University, Thanjvaur. 
International Journal of Hybrid Information Technology Vol.9, No.2 (2016) 